\title{
Introducing Complex Psychiatry
}

\author{
Joel Gelernter ${ }^{a, b}, c$ Renato Polimanti ${ }^{a}$ b \\ ${ }^{a}$ Department of Psychiatry, Yale University School of Medicine, West Haven, CT, USA; ${ }^{b}$ Department of Psychiatry, \\ Veterans Affairs Connecticut Healthcare System, West Haven, CT, USA; 'Departments of Genetics and Neuroscience, \\ Yale University School of Medicine, New Haven, CT, USA
}

"There was music in the cafés at night and revolution in the air" Tangled Up in Blue Nobel laureate Bob Dylan, 1975

With this issue, Molecular Neuropsychiatry transitions to new leadership under the name Complex Psychiatry. Why the name change? There are several reasons - we want to be explicit in our embrace of the complexity of current research in psychiatry - in complex trait genetics, the complex biology that underlies the genetics, indeed the whole range, in its difficult and fascinating vitality, of our research field. (And we also want to avoid any confusion with a certain other journal.) We will publish wellpowered studies, rigorous systematic reviews, and thought-provoking editorials. While respecting the highest quality criteria and the most appropriate data-sharing policies, we will offer rapid peer-review and rapid publication. We feel a strong need for this in the field. Besides the name change and the new editorial leadership, we usher in many new colleagues to the Editorial Board and as Associate Editors - already distinguished and some junior, intellectually diverse, and all enthusiastic. We are grateful to the previous editor, Bill Byerley, for his tireless

karger@karger.com

(C) 2020 S. Karger AG, Basel

www.karger.com/cxp

Karger work in establishing this journal in 2015 and moving it forward over the years.

The past 2 decades have been hugely consequential for neuropsychiatric research. The application of highthroughput technologies across numerous disciplines in psychiatry research has revolutionized our ability to investigate molecular and cellular processes in model organisms and in humans. There have been many consequences. As an example, the scientific community has been able to understand the development and the function of the brain structures better.

One of the best examples of how technological progress contributed to improve our understanding of the biology of psychiatric disorders is related to complex trait genetics. The first major genomewide association study (GWAS) was published only 15 years ago [1], but for psychiatry, it was 9 more years before revolution was really in the air [2]. We have gone from an era of candidate gene studies, most of which were completed in small samples and many of which were never really replicated, to the present era of well-powered GWAS with a strong record of replication. We can point to several reasons for this progress; care in ascertainment in smaller studies (what is small? A few thousand); the advent of large meta-ana- 
lytic consortia like the Psychiatric Genomics Consortium (PGC) [3] and large biobanks like the Million Veteran Program [4] and UK Biobank [5]; and the diverse set of powerful statistical tools that arose from these data $[6,7]$. We doubt if these developments could have been foreseen a decade ago by the most gifted prophet. Recognizing this progress and with it the movement of complex trait genetics into the mainstream of psychiatric research, we encourage journal submissions relevant to this research field.

While there has been huge scientific progress, the rapidly growing amount of information that has resulted so far has had only a very limited impact on evaluation and treatment of patients affected by psychiatric disorders. DSM-5 and ICD-10 diagnostic categories are mostly based on human experiential terms. Hundreds of psychiatric symptoms are aggregated into dozens of distinct diagnoses, which can be viewed as aggregating into 3 main domains (externalizing, internalizing, and psychosis) [8], which finally converge into a single dimension of general psychopathology ("p factor") [9]. The application of genetics and molecular and cellular biology to neuropsychiatric research has already helped investigators to move across these different phenotypic definitions and to begin to disentangle the mechanisms underlying this challenging complexity, with the ultimate goal of improving treatments.

Molecular Neuropsychiatry helped investigators share a number of studies addressing the complexity of neuropsychiatric traits with the scientific community. For this issue, we have selected 6 articles that represent the past contribution of Molecular Neuropsychiatry and delineate some of the new course of Complex Psychiatry.

In 2015, Altar and colleagues [10] provided initial evidence from 3 clinical studies supporting the utility of combinatorial pharmacogenomics-guided antidepressant therapy. Although pharmacogenomics has not yet delivered on its promise of personalized treatment for every patient, it is already helpful to a subset of patients receiving psychotropics; this article has been cited by 44 publications (so far) and has contributed to moving our field toward this application. Pharmacogenomics is increasing in utility and importance and is a major future direction for Complex Psychiatry.

In 2017, Crider and colleagues [11] presented the first evidence that the middle frontal gyrus in patients with autism spectrum disorder is affected by transcriptomic dysregulation of genes involved in the stress response of the endoplasmic reticulum. This study has been cited several times, including by a study published in Nature the following year [12].

Introducing Complex Psychiatry
Sedlak and colleagues [13] described findings from a clinical pilot study, suggesting that sulforaphane augments peripheral glutathione and affects brain metabolites that are altered in neuropsychiatric disorders. This study had a remarkable impact on the scientific community and the general media. According to Altmetric, this article received an attention score of 458 , which corresponds to the 99th percentile of all research outputs of the same age scored by Altmetric.

Among the review articles published in Molecular Neuropsychiatry, Cuperfain and colleagues [14] provided a comprehensive overview of the complex interaction of mitochondrial genetics and mitochondrial pathways in psychiatric disorders, with particular attention to the contribution of nuclear DNA, mitochondrial DNA, mitochondrial pathways, pseudogenes, nuclear-mitochondrial mismatch, and microRNAs to observable clinical phenotypes related to bipolar disorder and schizophrenia.

Another important review article was published by Dr. Dimitrios Avramopoulos [15] in 2018. This described recent advances in the genetics of schizophrenia with an overview of the different types of genetic variants identified (i.e., copy number variants, rare coding inherited and de novo variants, and numerous loci harboring common risk variants) and the emerging technologies (such as new methods in cell engineering) that could unlock the translational potential of these significant discoveries.

Finally, we include an article from Prevot and colleagues [16]. In 2019, they reported the synthesis of 4 novel imidazobenzodiazepine amide ligands, providing information regarding positive allosteric modulation, pharmacokinetic properties, and anxiolytic and antidepressant activities in adult mice. This study was noticed broadly in the scientific community with an Altmetric attention score of 131, which corresponds to the 98th percentile of all research outputs of the same age.

These articles illustrate some of the major developments in complex psychiatry in terms of data quality and quantity, and therefore biology. Something about what we should expect in the future - more data; more methods; better biology. But we do not dare guess what the scientific result of the availability of all of these data might be. We can confidently predict that it will be fun and exciting, and, together with our new Editorial Board, we will work hard to make Complex Psychiatry play an important role in bringing these developments to the scientific community. 


\section{Acknowledgements}

The authors thank Dr. Bill Byerley for establishing Molecular Neuropsychiatry, the members of the new editorial board of Complex Psychiatry, and Karger Publishers for their support.

\section{Disclosure Statement}

Drs. Gelernter and Polimanti are paid for their editorial work for Complex Psychiatry journal.

\section{Funding Sources}

The authors are supported by grants from the National Institutes of Health (R01DA012690, R01AA026364, U01MH109532, P50AA012870,R01DA037974,R21DA047527, andR21DC018098) and the Department of Veterans Affairs (1I01CX001849).

\section{Author Contributions}

J.G. and R.P. drafted, revised, and approved the manuscript.

\section{References}

1 Klein RJ, Zeiss C, Chew EY, Tsai JY, Sackler RS, Haynes C, et al. Complement factor $\mathrm{H}$ polymorphism in age-related macular degeneration. Science. 2005;308(5720):385-9.

2 Schizophrenia Working Group of the Psychiatric Genomics Consortium. Biological insights from 108 schizophrenia-associated genetic loci. Nature. 2014;511(7510):421-7.

3 Sullivan PF, Agrawal A, Bulik CM, Andreassen OA, Børglum AD, Breen G, et al. Psychiatric genomics: an update and an agenda. Am J Psychiatry. 2018;175(1):15-27.

4 Gaziano JM, Concato J, Brophy M, Fiore L, Pyarajan S, Breeling J, et al. Million veteran program: a mega-biobank to study genetic influences on health and disease. J Clin Epidemiol. 2016;70:214-23.

5 Collins R. What makes UK biobank special? Lancet. 2012;379(9822):1173-4.

6 Bulik-Sullivan BK, Loh PR, Finucane HK, Ripke S, Yang J, Patterson N, et al. LD Score regression distinguishes confounding from polygenicity in genome-wide association studies. Nat Genet. 2015;47(3):291-5.
7 Purcell SM, Moran JL, Fromer M, Ruderfer D, Solovieff N, Roussos $\mathrm{P}$, et al. A polygenic burden of rare disruptive mutations in schizophrenia. Nature. 2014;506(7487):185-90.

8 Caspi A, Moffitt TE. All for one and one for all: mental disorders in one dimension. Am J Psychiatry. 2018;175(9):831-44.

9 Caspi A, Houts RM, Belsky DW, GoldmanMellor SJ, Harrington H, Israel S, et al. The $p$ factor: one general psychopathology factor in the structure of psychiatric disorders? Clin Psychol Sci. 2014;2(2):119-37.

10 Altar CA, Carhart J, Allen JD, Hall-Flavin D, Winner J, Dechairo B. Clinical utility of combinatorial pharmacogenomics-guided antidepressant therapy: evidence from three clinical studies. Mol Neuropsychiatry. 2015;1(3): $145-55$.

11 Crider A, Ahmed AO, Pillai A. Altered expression of endoplasmic reticulum stress-related genes in the middle frontal cortex of subjects with autism spectrum disorder. Mol Neuropsychiatry. 2017;3(2):85-91.
12 Samant RS, Livingston CM, Sontag EM, Frydman J. Distinct proteostasis circuits cooperate in nuclear and cytoplasmic protein quality control. Nature. 2018;563(7731):407-11.

13 Sedlak TW, Nucifora LG, Koga M, Shaffer LS, Higgs C, Tanaka T, et al. Sulforaphane augments glutathione and influences brain metabolites in human subjects: a clinical pilot study. Mol Neuropsychiatry. 2018;3(4):21422.

14 Cuperfain AB, Zhang ZL, Kennedy JL, Gonçalves VF. The complex interaction of mitochondrial genetics and mitochondrial pathways in psychiatric disease. Mol Neuropsychiatry. 2018;4(1):52-69.

15 Avramopoulos D. Recent advances in the genetics of schizophrenia. Mol Neuropsychiatry. 2018;4(1):35-51.

16 Prevot TD, Li G, Vidojevic A, Misquitta KA, Fee C, Santrac A, et al. Novel benzodiazepinelike ligands with various anxiolytic, antidepressant, or pro-cognitive profiles. Mol Neuropsychiatry. 2019;5(2):84-97. 